\title{
Tesis doctorales sobre fotografía en la universidad española. Análisis de la producción y dirección (1976-2012)
}

\author{
Juan Miguel Sánchez-Vigil*, Juan Carlos Marcos-Recio**, María Olivera-Zaldua* \\ *Facultad de Ciencias de la Documentación, Universidad Complutense de Madrid. \\ Correo-e: jmvigil@ucm.es; molivera@ucm.es. \\ **Facultad de Ciencias de la Información. Universidad Complutense de Madrid. \\ Correo-e: jmarcos@ucm.es.
}

Recibido: 12-03-2013; $2^{a}$ version: 03-06-2013; $3^{a}$ version: 27-06-2013; Aceptado: 30-06-2013.

Cómo citar este artículo/Citation: Sánchez-Vigil, J. M.; Marcos-Recio, J. C.; Olivera-Zaldua, M. (2014). Tesis doctorales sobre fotografía en la universidad española. Análisis de la producción y dirección (1976-2012). Revista Española de Documentación Científica, 37(1):e034. doi: http://dx.doi.org/10.3989/redc.2014.1.1073

Resumen: Se analiza en este trabajo la producción y dirección de tesis doctorales sobre fotografía en la universidad española, es decir en todos los centros donde se imparten asignaturas relacionadas con la materia. El periodo de estudio se acota entre 1976 y 2012, y para el análisis se han aplicado técnicas bibliométricas. Se ha empleado como fuente principal la base de datos Teseo del Ministerio de Educación, cuya información se ha contrastado y completado con la hemeroteca Dialnet, el catálogo Cisne, la Red de Bibliotecas Universitarias y el Centro Virtual Cervantes. El resultado ha sido de 275 tesis, 39 de ellas codirigidas. Se describen la cuantificación, la temática, la distribución por universidades, centros y departamentos, así como el número de directores y codirectores. Se contextualiza el tema con una introducción sobre la incorporación de la fotografía a los estudios oficiales y universitarios.

Palabras clave: Dirección de tesis doctorales; documentación fotográfica; fotografía; tesis doctorales; producción científica; universidad.

Doctoral theses on photography in Spanish universities. Analysis of production and supervision (1976-2012)

Abstract: This paper analyses the production and supervision of doctoral theses on photography in Spanish universities, i.e., in all centres where courses related to this subject matter are taught. The period of study runs from 1976 until 2012, and we have applied bibliometric techniques to conduct this analysis. We have used as the main source of information the Teseo database of the Ministry of Education, whose information has been contrasted and completed with the Dialnet virtual library, the Cisne catalogue, the Network of University Libraries and the Centro Virtual Cervantes. In all, 275 theses were identified, of which 39 had been co-directed. The results describe the quantification, the topic, the distribution by universities, centres and departments, and the number of directors and co-directors. By way of contextualization, there is an introduction into the incorporation of photography into official and university studies.

Keywords: Direction of doctoral theses; photographic documentation; photography; doctoral theses; scientific production; university.

Copyright: ( 2014 CSIC. Este es un artículo de acceso abierto distribuido bajo los términos de la licencia Creative Commons Attribution-Non Commercial (by-nc) Spain 3.0. 


\section{INTRODUCCIÓN}

La fotografía se relaciona generalmente con los estudios de comunicación audiovisual o bellas artes, sin embargo, sus tres valores principales, artístico, informativo y documental, le confieren un carácter transversal y por tanto la vinculan a distintas áreas de conocimiento: ciencias, arte, humanidades o ciencias sociales (Sánchez Vigil, 2006). Gisèle Freund caracterizó la fotografía como el medio de expresión de la sociedad, establecida sobre la civilización tecnológica, con poder para reproducir exactamente la realidad externa (Freund, 1999).

La cualidad transversal de la fotografía la aproxima a un sinfín de temas de investigación, por lo que basta con analizar los títulos de las tesis publicadas para observar la diversidad de enfoques, desde el teórico hasta el creativo: identificación, literatura, biografías, semiótica, técnica, etc. Por otra parte, la transversalidad hace que su clasificación sea diversa: empírica, estética, y en cualquier caso exterior al objeto; de ahí que Roland Barthes la presentara como inclasificable por el hecho de que "no hay razón para marcar una de sus circunstancias en concreto" (Barthes, 1994).

La aplicación de la fotografía en España a los estudios oficiales se realizó por Real Decreto de 31 de mayo de 1904, tras la reforma del programa de la Escuela Superior de Guerra que incluyó por primera vez la disciplina en la enseñanza pública y privada. En 1916, por Real Orden del Ministerio de Orden Público y Bellas Artes, se creó en Granada la primera Escuela Oficial de Fotografía dirigida por Manuel Torres Molina, sin embargo, hasta 1953 no se consideró asignatura universitaria, cuando la Escuela Oficial de Periodismo la ofreció en el I Curso de Periodistas Gráficos. Un año más tarde, en 1954, el historiador Beaumont Newhall impartía en la Universidad de Rochester las primeras clases de fotografía artística (Riego, 1993).

Por lo que respecta a la universidad española, la fotografía ha sido mayor objeto de estudio en las facultades de Arte y Comunicación. Las primeras facultades de Ciencias de la Información se crearon en nuestro país en 1971, en la Universidad Complutense de Madrid y en la Autónoma de Barcelona (Embid, 2002), y las de Bellas Artes abrieron su puertas en 1978 por transformación de las antiguas Escuelas Superiores. La primera tesis sobre fotografía en el periodo que nos ocupa fue de carácter técnico, Fotometría fotográfica, leída en la Facultad de Físicas de la Universidad Complutense, el 19 de noviembre de 1976, por José María García-Pelayo Echevarría, bajo la dirección de José María Torroja Menéndez.

Entre 1976, año del cambio político, y la actualidad, la evolución de la fotografía en España ha sido extraordinaria. Además de la incorporación de la fotografía como disciplina a los estudios de comunicación, documentación, arte e historia, se han fundado numerosas asociaciones, escuelas y centros auspiciados tanto por el Estado como por las Comunidades Autónomas y los Ayuntamientos. Por otra parte la creación de nuevos diarios, El País y El Mundo entre otros, ha dado a la imagen fotográfica un valor que hoy es objeto de estudio. En 1983 Joan Fontcuberta criticó las deficiencias metodológicas de la historia de la fotografía, señalando como una de las causas "la endeblez y anquilosamiento de la estructura investigadora de la universidad" (Fontcuberta, 1983). Cuatro años después, en 1987, Bernardo Riego puso en marcha el Aula de Fotografía en la Universidad de Cantabria supervisado por el Vicerrectorado de Extensión Universitaria, y en 1993 ya se estudiaba en las facultades de Bellas Artes, Historia del Arte, Ciencias de la Información, y con mayor especialización en Ciencias de la Educación, Ciencias del Mar y Geografía e Historia.

Tras la implantación del Espacio Europeo de Educación Superior (Declaración de Bolonia), por el que se pusieron en marcha los nuevos estudios de Grado y Máster (Real Decreto del Ministerio de Educación y Ciencia 1393/2007 de 29 de octubre), la fotografía ha sido incorporada como asignatura obligatoria en varios másteres, por ejemplo, en Gestión de la Documentación y Bibliotecas de la Facultad de Ciencias de la Documentación de la Universidad Complutense de Madrid (Medios de Comunicación $)^{1}$, o en el de Patrimonio Audiovisual de la Facultad de Ciencias de la Información, de la misma Universidad, iniciado en el curso 20122013, que incluye cuatro asignaturas en el progra$\mathrm{ma}^{2}$. En este sentido es importante señalar el interés por la recuperación del patrimonio fotográfico tanto por las instituciones nacionales como internacionales (Moreiro, 2010).

Los estudios sobre tesis doctorales tienen referente en bibliometría en las investigaciones de Delgado, Torres, Jiménez y Ruiz-Pérez (2006), y en el observatorio IUNE, creado para el seguimiento de la actividad investigadora en la universidad española (Sanz Casado y otros, 2011). También desde el punto de vista del control y acceso bibliográfico (Moralejo, 2000; Fuentes y Arguimbau, 2010), así como de manera específica en campos como comunicación (Jones y Barí, 2000), publicidad (Marcos Recio, Martínez Pestaña y Blasco López, 2012), radio y televisión (Repiso, Torres, Delgado, 2011a, b), biblioteconomía y documentación (López Yepes, 2008), cine (Repiso, Torres, Delgado, 2007), relaciones públicas (Castillo y Xifra, 2006), o información y documentación (Fuentes Pujol y González Quesada, 2002). Sin embargo, no se han realizado hasta ahora investigaciones sobre tesis doctorales de fotografía en la universidad española, si bien disponemos de algunas referencias generales (Riego, 1993; Vega, 2007; Vázquez, 2008).

Es objeto de este trabajo conocer la producción y dirección de tesis doctorales sobre fotografía en las universidades españolas en el periodo comprendi- 
do entre 1976 y 2012, enmarcando los resultados en el amplio espectro de la Documentación Fotográfica. Se pretende cuantificar las tesis, valorar su evolución en cuanto a la producción, establecer las temáticas específicas, averiguar la distribución por universidades, centros y departamentos, y señalar la aportación de los directores y codirectores de las mismas.

\section{METODOLOGÍA}

El estudio, como se ha indicado, comprende el periodo 1976-2012, coincidente con la apertura de las Facultades de Ciencias de la Información de la UCM y la Universidad de Barcelona, y cuyas primeras tesis fueron leídas, por tanto, en la segunda parte de los setenta. El estudio se ha realizado principalmente a partir de los contenidos de la base de datos Teseo, del Ministerio de Educación, Cultura y Deporte (www.mcu.es/teseo), que recoge las tesis depositadas por las universidades, completadas con las fuentes secundarias que se indican: Dialnet (http:// dialnet.unirioja.es), catálogo Cisne de la Biblioteca de la Complutense (cisne.sim.ucm. es), Red de Bibliotecas Universitarias (http:// rebiun.absysnet.com) y el centro virtual Cervantes (www.cervantesvirtual.com). Fuente secundaria de gran interés ha sido el libro de Jones y Barí (2000), donde se recogen 45 tesis sobre fotografía de las que 8 no se localizan en Teseo.

Teseo permite buscar por título o resumen, o bien por ambos campos, si bien tiene limitaciones como fuente, ya que no todas las tesis se encuentran en esta base de datos, y además, la información no es completa, con ausencia de datos como fecha exacta de lectura, departamentos o facultades. Asimismo presenta problemas de normalización en cuanto a nombres de personas e instituciones, por lo que ha sido necesario contrastarlos y completarlos.

Con el fin de conseguir el mayor número de referencias se han hecho búsquedas en Teseo con varios términos, partiendo del prefijo foto* y completando la investigación con la búsqueda por otros descriptores: cámara, prensa, retrato, ilustración, fototipia, reportero, reportaje, daguerrotipo, fotograbado, fotomecánica y fotoperiodismo. Los resultados han sido desiguales, así del término ilustración resultan 22 tesis, de las que solo 4 son de fotografía, y de prensa, 515 registros, de los que también solo 4 son sobre prensa gráfica.

Se ha elaborado una base de datos relacional donde se ha volcado toda la información a partir de la cual se han obtenido las referencias del autor, título, fecha de lectura, universidad, facultad o centro, departamento, director y descriptores. También se ha realizado una intensa labor documental para completar los datos que faltaban en Teseo, fundamentalmente nombres de directores y facultades. Los primeros resultados a partir de la clave fotograf* fueron 1138 registros, ya que el prefijo foto (del griego phos: luz) se aplica a es- tudios relacionados con varias ciencias (medicina, construcción, arquitectura, etc.). La búsqueda por universidades dio como resultado 713 trabajos en 78 centros, cuyos contenidos fueron revisados uno a uno y finalmente, quedaron reducidos a $267 \mathrm{co}-$ rrespondientes a 45 centros, más los 8 localizados en Jones y Barí (2000).

El uso de términos genéricos en la base de datos para la descripción de contenidos, en lugar de palabras clave específicas en cada caso, dificulta la recuperación de la información. Por otra parte, los descriptores no se corresponden con los contenidos en numerosos casos, incluso cuando el término fotografía o similar figura en el título. Indicamos como ejemplo los siguientes títulos: Imagen de la mujer en el fondo fotográfico de la guerra civil española de la biblioteca nacional. Madrid, 1936-1939, de Beatriz de las Heras Herrero, dirigida por Antonio Rodríguez de las Heras y Retratos fotográficos postmorten en Galicia (siglos XIX y XX), de Virginia de la Cruz, dirigida por Estrella de Diego, cuyo descriptor es solo Historia; Fotografía en Colombia en los años 70, de Santiago Rueda Fajardo, dirigida por Lourdes Cirlot Valenzuela, cuyo único descriptor es Historia del Arte, y La colección iconográfica del compositor Joaquín Turina. Análisis documental: inventario y catalogación, de María Olivera Zaldua, dirigida por Juan Miguel Sánchez Vigil, cuyo único descriptor es Lingüística. Por ello, ha sido necesario revisar todos los resúmenes para comprobar la relación directa de la investigación con la fotografía.

Las fuentes complementarias se han utilizado para la localización y comprobación de fechas, departamentos, facultades $y$, en su caso, los nombres de los autores y directores. Asimismo se hizo una búsqueda en Dialnet de tesis doctorales, que se contrastaron con las localizadas en Teseo. La información contenida en Dialnet se refiere a autores, directores, centros, año de lectura y resumen del contenido, por tanto, sin descriptores. El resto de fuentes se han utilizado para completar aquellos datos no localizados ni en Teseo ni en Dialnet. Por otra parte, se ha contactado directamente, en algunos casos, con los centros y departamentos, así como con los propios doctores y/o con los directores, siempre con el fin de completar la información.

Una vez obtenida toda la información, se han establecido categorías de las tesis por contenidos, y, tras el proceso de los datos, se han logrado los resultados que figuran a continuación: producción global, temáticas, aportación de las universidades, centros y departamentos, directores y codirecciones. Se incluye finalmente un anexo con las tesis por año, nombre del director, universidad y autor.

\section{RESULTADOS}

\subsection{Producción global de tesis}

Los factores que determinan las tesis durante el proceso de creación, evaluación y difusión son va- 
rios, desde el tema de estudio, el director o la línea de investigación, hasta el departamento o el centro. Establecemos, en primer lugar, la producción global y su evolución en el tiempo (Figura 1).

El total de tesis resultantes es de 275 entre 1976 y 2012 , con una media anual de 7,63 y una clara tendencia al alza. Se observa que en los dos primeros años (1976-1977) solo se presentaron 3 trabajos, y que se produjo un vacío hasta 1981. A partir de 1983, la evolución fue constante hasta alcanzar las 21 tesis a finales de la década de los ochenta, pasando a 66 en los noventa, y a 141 en la del 2000. El año de mayor número de tesis fue 2004 con 21, seguido de 2012 con 20, 2001 con 19 y 2010 con 17 trabajos, mientras que solo se leyó una en los años 1976, 1980, 1983 y 1984. Las mayores variaciones al alza se produjeron en los años 1989 con 6 tesis, 1994 con 8, 1998 con 13, 2003 con 17, y 2004 con 21.

En el año 2006 se produjo un considerable descenso hasta 9 tesis, y tras una importante recuperación en 2007 y 2008, con 12 y 17 investigaciones respectivamente, hubo un nuevo descenso hasta 10 en 2009. En los últimos tres años, el incremento ha sido constante, con 17 tesis en 2010, 19 en 2011 y 20 en 2012. En general, el periodo de mayor concentración corresponde a los años 20072012, con 95 trabajos, es decir el 35,58\% del total.

El aumento producido en los años 2002-2003 podemos relacionarlo con la creación de centros de documentación fotográfica en las Comunidades Autónomas, así como con la puesta en marcha de eventos internacionales como PHotoEspaña, creado a finales de los noventa y en pleno auge en esos años. El segundo periodo de aumento de tesis (2010-2012), coincide con la aplicación del Espacio Europeo de Educación Superior (EEES), a partir del Real Decreto de 29 de octubre de 2007, que estructuró los nuevos títulos en Grado, Máster y Doctorado. La modificación de los planes de enseñanza ha propiciado y fomentado la incorporación de nuevas asignaturas sobre la materia, fundamentalmente en los másteres. Son ejemplo el Máster en Gestión de Bibliotecas y Documentación de la Facultad de Ciencias de la Documentación de la UCM, con una especialidad en Medios de Comunicación, o el de Patrimonio Audiovisual de la Facultad de Ciencias de la Información de la misma universidad, con especialidad en Fotografía.

\subsection{Temáticas}

Con el fin de establecer los principales temas de las tesis, se han tenido en cuenta los descriptores de Teseo para la recuperación de contenidos, que ascienden a 150 en las 275 investigaciones, destacando por la cantidad de asignaciones las veinte palabras clave que se indican a continuación: fotografía (110 referencias), ciencias de las artes y de las letras (96), teoría, análisis y crítica de las bellas artes (96), historia del arte (36), historia (35), historias especializadas (24), pintura y estética de las bellas artes (18), dibujo y grabado (16), opinión pública e historia del arte de los siglos xIx y xx (12), ciencias políticas (11), prensa (10), arquitectura (9), lingüística (8), óptica fotográfica, historia del periodismo, documentación y cinematografía (7). Con menos de seis referencias figuran los 130 descriptores restantes.

Una vez analizados los contenidos en detalle, se han establecido siete categorías generales (Tabla I): Arte, Autores (vida y obra), Comunicación, Documentación, Historia, Sociología, y Técnica y Tecnología. En el apartado Documentación se han incluido las tesis sobre este tema más aquellas referidas a fondos y colecciones, y al uso específico de la foto en la investigación, y en el de Sociología se han agrupado los temas sobre esta materia además de los relacionados con su aplicación a la educación, psicología, turismo o derecho.

Figura 1. Evolución de la producción de tesis por años

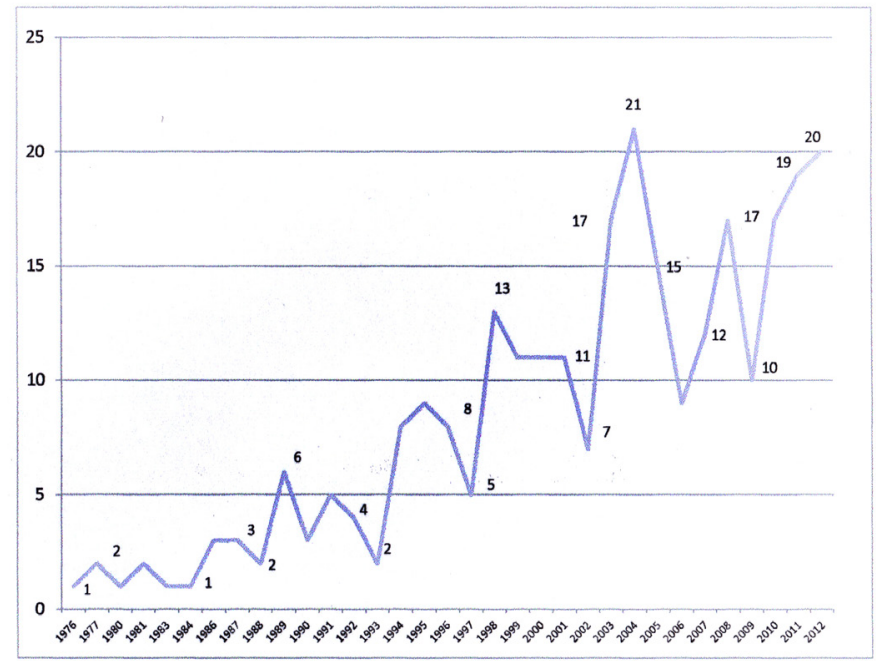




\subsection{Producción por universidades}

Las universidades españolas que cuentan con tesis doctorales sobre fotografía son 45 , si bien en tan solo 17 centros se han leído 5 o más trabajos (Tabla II). En primer lugar figura la Universidad
Complutense de Madrid con 49 títulos, lo que supone el $17,81 \%$ del total, porcentaje ciertamente significativo; le siguen la Universitat de Barcelona con $26(9,45 \%)$, la Politécnica de Valencia con 22 $(8,23 \%)$ y la del País Vasco con 21 (7,63\%).

Tabla I. Temática general de los contenidos de las tesis

\begin{tabular}{lc}
\hline \multicolumn{1}{c}{ Categoría } & No de tesis \\
\hline Arte & 76 \\
\hline Autores (vida y obra) & 38 \\
\hline Comunicación & 39 \\
\hline Documentación & 41 \\
\hline Historia & 17 \\
\hline Sociología & 28 \\
\hline Técnica y Tecnología & 36 \\
\hline Total & 275 \\
\hline
\end{tabular}

Tabla II. Producción de tesis por universidades

\begin{tabular}{|c|c|c|c|}
\hline No de tesis & $\%$ & & Universidad \\
\hline 49 & 17,81 & Universidad Complutense de Madrid & \\
\hline 26 & 9,45 & Universitat de Barcelona & \\
\hline 22 & 8,23 & Universitat Politècnica de Valencia & \\
\hline 21 & 7,63 & Universidad del País Vasco & \\
\hline 12 & 4,49 & Universidad de Salamanca & \\
\hline 11 & 4,11 & Universidad La Laguna & \\
\hline 10 & 3,74 & Universidad de Navarra & \\
\hline 9 & 3,37 & Universidad de Granada & \\
\hline 8 & 2,99 & $\begin{array}{l}\text { Universidad de Sevilla } \\
\text { Universitat de Valencia }\end{array}$ & Universitat Autónoma de Barcelona \\
\hline 7 & 2,62 & Universidad de Castilla-La Mancha & Universidad Politécnica de Madrid \\
\hline 6 & 2,24 & Universidad de Vigo & \\
\hline 5 & 1,87 & $\begin{array}{l}\text { Universidad Jaume I de Castellón } \\
\text { Universidad de Málaga }\end{array}$ & U. Nacional de Educación a Distancia \\
\hline 4 & 1,49 & $\begin{array}{l}\text { Universidad Autónoma de Madrid } \\
\text { Universidad Carlos III de Madrid } \\
\text { Universidad de Murcia }\end{array}$ & $\begin{array}{l}\text { Universidad Politècnica de Catalunya } \\
\text { Universidad San Pablo-Ceu }\end{array}$ \\
\hline 3 & 1,12 & $\begin{array}{l}\text { Universidad de Alicante } \\
\text { U. Miguel Hernández de Elche } \\
\text { Universitat Pompeu Fabra }\end{array}$ & $\begin{array}{l}\text { Universidad de Zaragoza } \\
\text { Universidad de Santiago de Compostela }\end{array}$ \\
\hline 2 & 0,74 & $\begin{array}{l}\text { Universidad de A Coruña } \\
\text { Universidad de Cádiz }\end{array}$ & Universitat Rovira i Virgili \\
\hline 1 & 0,37 & $\begin{array}{l}\text { Universidad de Cantabria } \\
\text { Universidad Católica San Antonio } \\
\text { Universidad de Extremadura } \\
\text { Universitat de Girona } \\
\text { Universitat de les Illes Balears } \\
\text { Universidad de Jaén } \\
\text { Universidad de León } \\
\text { Universitat de Lleida }\end{array}$ & $\begin{array}{l}\text { Universitat Oberta de Catalunya } \\
\text { Universidad de Oviedo } \\
\text { Universidad de Palmas de Gran Canaria } \\
\text { Universidad Pública de Navarra } \\
\text { Universidad Ramón Llull } \\
\text { Universidad Rey Juan Carlos } \\
\text { Universidad de Valladolid }\end{array}$ \\
\hline 275 & & 45 Universidades & \\
\hline
\end{tabular}


Se observa que la mayor producción surge de las universidades, centros y departamentos históricos, con Bellas Artes y Comunicación a la cabeza. No se advierten picos destacables en la producción, por tanto se puede calificar de regular en todas las universidades. En el caso de la Politécnica de Valencia se advierte un importante número de tesis leídas, procedentes en su mayoría de la Facultad de Bellas Artes (21 de 22), con una media de una o dos por año, si bien, de seis departamentos diferentes. En general se ha mantenido una media constante en casi todos los departamentos, y no se prevé un cambio radical en breve. Entre las nuevas especialidades, la Documentación ha aportado 15 nuevas tesis entre 2003 y 2012 desde cinco universidades: Granada, Carlos III de Madrid, Complutense, Murcia y Politècnica de Valencia, lo que viene a confirmar que se suma al resto de centros históricos pero manteniendo la regularidad general.

\subsection{Aportación de las Facultades y Departa- mentos}

El número de Departamentos en los que se han leído tesis sobre fotografía en el periodo estudiado es de 114, distribuidos en 47 facultades (Figura 2). Se han establecido cuatro grupos generales: Ciencias, Bellas Artes, Humanidades y Ciencias Jurídicas, Comunicación y Ciencias Sociales. En las facultades de Bellas Artes se han leído 101 tesis $(36,73 \%)$, en Comunicación $79(28,73 \%)$, en Humanidades $63(22,90 \%)$ y en Ciencias 32 $(11,63 \%)$. (Figura 2$)$

De las 101 tesis presentadas en las facultades de Bellas Artes, 25 corresponden a la Universidad Complutense de Madrid, 21 a la Politècnica de Valencia, 21 a la de Barcelona, 8 a la de Salamanca, 8 a la del País Vasco, 7 a la de La Laguna, 4 a Granada, 3 a Sevilla y 2 a las facultades Miguel Hernández de Elche y Vigo.

La terminología para referirse a las facultades de Comunicación, Información y Ciencias Sociales es diversa, pero sus contenidos están perfectamente definidos. El centro con mayor número de tesis es la Facultad de Ciencias de la Información de la Universidad Complutense de Madrid con 20 trabajos, en segundo lugar se encuentra la de Ciencias Sociales y de la Comunicación de la Universidad del País Vasco con 11, seguida de Ciencias de la Comunicación de la Universidad de Navarra con 6, y de siete facultades con 4 trabajos: Ciencias Sociales y de la Comunicación de Vigo, Humanidades y Ciencias de la Comunicación de San Pablo-Ceu, Humanidades, Comunicación y Documentación de la Universidad Carlos III de Madrid, Ciencias de la Comunicación de Sevilla, y Ciencias Humanas y Sociales de la Jaume I de Castellón. En el resto de facultades se presentaron tres o menos tesis.

Por lo que respecta a los centros de Humanidades, la Facultad de Letras de la Universidad de Castilla La Mancha figura en primer lugar con 7 tesis, y en un segundo bloque un grupo de tres con 4 trabajos: Geografía e Historia de la Universidad de Barcelona, Filosofía y Letras de la Autónoma de Madrid, y Filosofía y Letras de Navarra. Los resultados indican 3 tesis para la Facultad de Geografía e Historia de la Universidad Nacional de Educación a Distancia, más las de Filosofía y Letras de Zaragoza y Málaga. Con 2 tesis figuran las facultades de Humanidades de la Pompeu Fabra, la de Letras de la Rovira Virgili, Filosofía de Barcelona, Ciencias de la Educación de Valencia, y Filosofía y Letras de Cádiz. Con una sola tesis aparecen las facultades de Derecho, Educación, Psicología, Filología, Ciencias del Turismo, Ciencias del Deporte y la Educación Física, y Ciencias Jurídicas y Económicas.

En cuanto a las facultades de Ciencias se produce un equilibro, con 32 tesis leídas en 28 centros. Tan solo la Facultad de Informática de Murcia cuenta con 3 tesis, y con 2 la de Arquitectura de la Politécnica de Madrid y las de Físicas de la Complutense y Valencia. Por tanto, son 24 las facultades con tan solo una tesis presentada.

Por lo que respecta a los departamentos, destacan por su producción los de Historia del Arte con 44 tesis, seguidos de los de Comunicación e Información con 41, Dibujo y Expresión Artística con 48, Diseño e Imagen con 14, Periodismo con 12, Biblioteconomía y Documentación con 9, Filosofía con 6 y Humanidades con 5 . El resto se encuentra por debajo de las 4 tesis.

En lo que se refiere al Arte, el Departamento de Historia del Arte y Bellas Artes de la Universidad de Salamanca encabeza la relación con 8 tesis, seguido por los de Historia del Arte de las universidades de Castilla-La Mancha con 7 y La Laguna con 4. Los departamentos de Historia del Arte de Barcelona, Zaragoza y Nacional de Educación a Distancia suman 3.

En Comunicación y Ciencias de la Información son los departamentos de Comunicación Audiovisual los que reúnen mayor número de trabajos, que ascienden a 31 , siendo el primero el de Comunicación Audiovisual y Publicidad I de la Complutense con 11, el segundo, el de la Universidad del País Vasco con 8 trabajos, y el tercero, el de Comunicación Audiovisual, Documentación e Historia del Arte de la Politécnica de Valencia con 4 investigaciones. Con 3 tesis aparece Comunicación Audiovisual y Nuevas Tecnologías. En cuanto a los departamentos de Periodismo, las tesis presentadas sobre fotografía son 11, destacando con 3 los de las Universidades de País Vasco, Sevilla y Complutense, más dos en la Autónoma de Barcelona y una en Málaga.

Los departamentos de Biblioteconomía y Documentación aportan 9 tesis: 3 en la Facultad de Informática de Murcia, 2 en la Universidad Complutense de Madrid, 2 en la Carlos III de Madrid y otras 2 en la de Granada. Por último, las tesis leídas en los departamentos de Humanidades son 
5, distribuidas entre la Pompeu Fabra (2), Carlos III de Madrid (2) y Alicante (1), y las presentadas en Filosofía ascienden a 6: Autónoma de Madrid y Barcelona, Universitat de Valencia, Complutense, Nacional de Educación a Distancia y Jaume I de Castellón.

\subsection{Directores de tesis}

Los profesores que han dirigido las 275 tesis son 251 (incluidos 40 codirectores), número elevado que indica diversidad y que tiene como consecuencia que solo 13 profesores hayan dirigido más de 2 tesis en el periodo de estudio (Tabla III). Los directores más productivos son: Joaquín Perea González en la Universidad Complutense de Madrid con 7 tesis (una codirigida) en la Facultad de Bellas Artes, seguido de Román de la Calle con 6 (4 codirigidas) en las universidades de Valencia ( 1 en la Facultad de Filosofía y Ciencias de la Educación, y 1 en Geografía e Historia), Politècnica de Valencia ( 3 en la Facultad de Bellas Artes) y 1 en Vigo (Facultad de Ciencias de la Comunicación).

María Dolors Tapias Gil y Francisco Caja López han dirigido 5 cada uno en la Facultad de Bellas Artes de la Universidad de Barcelona, Javier Marzal Felici 4 en Ciencias Humanas y Sociales de la Universidad Jaume I de Castellón (una codirigida), y Antonio Lara García 3 en la Facultad de Ciencias de la Información de la UCM y 1 en Geografía e Historia de la misma universidad. Margarita Ledo ha dirigido 4 en las Facultades de Ciencias de la Comunicación de la Autónoma de Barcelona (2) y de la Universidad de Santiago (2).

La mayoría de los directores de tesis son Catedráticos o Profesores Titulares, vinculados a los departamentos y centros históricos. Se constata también su prestigio en la especialidad $y$, por tanto, su pertinencia. La diversidad es otra de las conclusiones, ya que de 251 directores, 25 han dirigido dos tesis y 213 solo una.

\subsection{Codirecciones}

El sistema de trabajo en codirección comenzó a aplicarse a finales de los años noventa del siglo pasado. En el caso que nos ocupa todas las tesis han sido codirigidas por dos profesores, con excepción de una con tres, leída en la Universidad de Alicante el 30 de abril de 2010 por Raquel Pérez del Hoyo. El número de tesis codirigidas asciende a 39 (Tabla IV), es decir el 14,44\%. La primera se presentó el 8 de noviembre de 1999 en la Universidad de Vigo por el Departamento de Expresión Artística, codirigida por los profesores Yolanda Herranz Pascual y Román de la Calle. Desde entonces se observa un aumento progresivo que responde a estos parámetros: entre el año 2000 y el 2005 la media fue de 2 , aumentó a 4 en 2007-2009, a 5 en 2010-2011, y a 7 en 2012. Son excepciones los años 2006 con solo una, y 2009 en el que no se codirigió ninguna.

Figura 2. Tesis por especialidades en Facultades y Departamentos

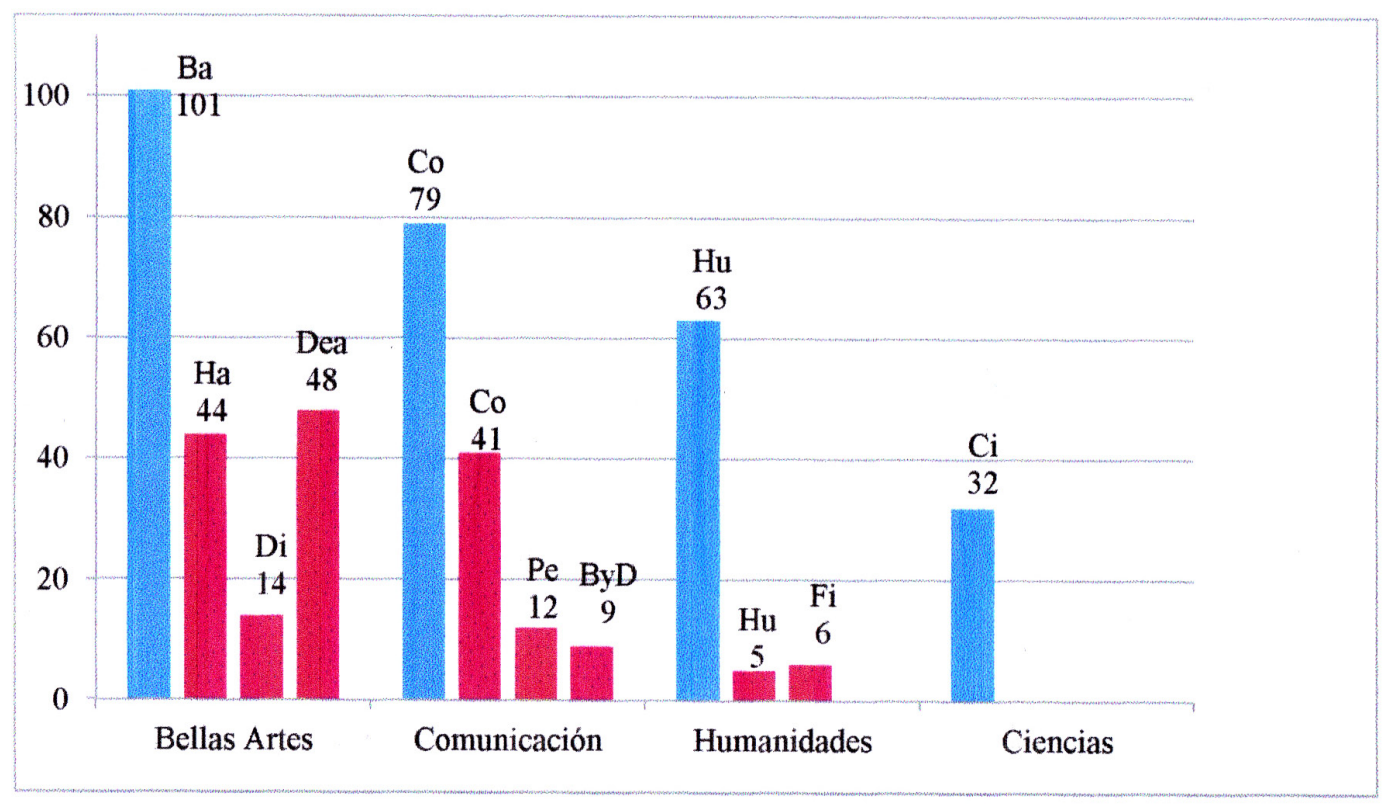

Facultades: Ba (Bellas Artes); Co (Comunicación); Hu (Humanidades); Ci (Ciencias)

Departamentos: Ha (Ha del Arte); Co (Comunicación); Dea (Dibujo y Expresión Artística); Di (Diseño e Imagen); Pe (Periodismo); ByD (Biblioteconomía y Documentación); Fi (Filosofía); Hu (Humanidades) 
Se observa una gran cantidad y diversidad de centros en los que se han codirigido tesis, ya que son 25 las universidades, y a la cabeza de ellas la Politècnica de Valencia con 5 trabajos entre 2001 y 2010, seguida de la Complutense con 3 en 2004, 2007 y 2008, y de Sevilla, con el mismo número en
2000, 2002 y 2012. Las universidades con 2 tesis codirigidas son Salamanca (2006 y 2011), La Laguna (2010 y 2012), Murcia (2002 y 2008), Granada (2002 y 2012), Politècnica de Catalunya (2001 y 2011), y Universitat de Valencia (2005 y 2008).

Tabla III. Directores de tesis de fotografía

\begin{tabular}{|c|c|c|c|c|}
\hline Director & Tesis & Universidad & Facultad & $\%$ \\
\hline Perea González, J. & 7 & Complutense & Bellas Artes & 2,61 \\
\hline Calle, Román de la & 6 & $\begin{array}{l}\text { Universidad Valencia (2) } \\
\text { Politècnica Valencia (3) } \\
\text { Vigo (1) }\end{array}$ & $\begin{array}{l}\text { Filosofía y CC. Educación (1) Geografía } \\
\text { e Historia (1) } \\
\text { Bellas Artes } \\
\text { CC Comunicación }\end{array}$ & 2,24 \\
\hline Tapias Gil, M. D. & 5 & Universidad de Barcelona & Bellas Artes & 1,87 \\
\hline Caja López, F. & 5 & Universidad de Barcelona & Bellas Artes & 1,49 \\
\hline Marzal Felici, J. & 4 & Jaume I de Castellón & CC Humanas y Sociales & 1,49 \\
\hline Lara García, A. & 4 & Complutense & $\begin{array}{l}\text { CC de la Información (3) } \\
\text { Geografía e Historia (1) }\end{array}$ & 1,49 \\
\hline Ledo Andino, M. & 4 & $\begin{array}{l}\text { Santiago Compostela (2) } \\
\text { Autónoma Barcelona (2) }\end{array}$ & $\begin{array}{l}\text { CC de la Comunicación } \\
\text { CC de la Comunicación }\end{array}$ & 1,49 \\
\hline Aliaga Morell, J. & 3 & Politècnica de Valencia & Bellas Artes & 1,12 \\
\hline Carbó Perseguer, E. L. & 3 & Barcelona & Bellas Artes & 1,12 \\
\hline Esparza Estaún, J.R. & 3 & País Vasco & CC Sociales e Información & 1,12 \\
\hline García Melero, J. E. & 3 & UNED & Geografía e Historia & 1,12 \\
\hline Prado Pico, E. & 3 & $\begin{array}{l}\text { Autónoma Barcelona (1) } \\
\text { País Vasco (2) }\end{array}$ & $\begin{array}{l}\text { CC de la Comunicación } \\
\text { CC Sociales e Información }\end{array}$ & 1,12 \\
\hline Vázquez Medel, M. A. & 3 & Sevilla & $\begin{array}{l}\text { Filología (1) } \\
\text { Comunicación (2) }\end{array}$ & 1,12 \\
\hline 25 directores & 2 & & & 0,74 \\
\hline 213 directores & 1 & & & 0,37 \\
\hline 251 directores & 275 & & & \\
\hline
\end{tabular}

Tabla IV. Tesis codirigidas en las universidades

\begin{tabular}{|c|c|c|c|}
\hline Universidad & No de tesis & Universidad & No de tesis \\
\hline Universitat Politècnica de Valencia & 5 & Universitat Rovira i Virgili & 1 \\
\hline Universidad de Sevilla & 3 & Universidad Rey Juan Carlos & 1 \\
\hline Universidad Complutense de Madrid & 3 & Universidad de Málaga & 1 \\
\hline Universidad de Salamanca & 2 & Universitat de Lleida & 1 \\
\hline Universitat Politècnica de Catalunya & 2 & Universitat Autónoma de Barcelona & 1 \\
\hline Universidad de Murcia & 2 & Universidad Jaume I de Castellón & 1 \\
\hline Universidad de Granada & 2 & Universitat de Girona & 1 \\
\hline Universidad de La Laguna & 2 & Universidad de Castilla-La Mancha & 1 \\
\hline Universitat de Valencia & 2 & Universidad Carlos III de Madrid & 1 \\
\hline Universidad de Zaragoza & 1 & Universidad Autónoma de Madrid & 1 \\
\hline Universidad de Vigo & 1 & Universidad de Alicante & 1 \\
\hline Universidad de Santiago de Compostela & 1 & Universidad de A Coruña & 1 \\
\hline Universidad San Pablo-Ceu & 1 & & \\
\hline
\end{tabular}


Del análisis del estudio resulta que no hay una concentración de tesis codirigidas en ninguna universidad. Lo mismo sucede en lo que se refiere a los departamentos, puesto que solo en cuatro del total se codirigen tesis: Dibujo II (Bellas Artes de la UCM), Información y Documentación (Informática de Murcia), Periodismo (Comunicación de Sevilla) y Pintura (Bellas Artes de la Politècnica de Valencia). Por otra parte, en lo que respecta al profesorado solo un docente repite codirección en su departamento (Informática y Documentación de la Universidad de Murcia). Analizando los títulos y resúmenes de contenidos, se justifica en la mayoría de los casos, la codirección por la combinación de disciplinas. Así, por ejemplo, las cuatro tesis de la Politècnica de Valencia relacionan la fotografía con la pintura, la prensa, la informática o las artes gráficas; las tres tesis de la Universidad de Granada con la cartografía, la arquitectura y la educación, y las tres de la Complutense con el arte (2) y la pedagogía (1).

El profesor Ramón de la Calle figura en primer lugar con 4 tesis codirigidas, dos de ellas en la Politècnica de Valencia junto a Pedro Esteban Fernández (2003) y Joan Aliaga Morell (2004), una en Vigo junto a Yolanda Herranz Pascual (1999), y la cuarta en la Universitat de Valencia con Pilar Pedraza Martínez (2005). Le siguen con 2 tesis codirigidas los profesores Joaquim Regot Marimón y José Vicente Rodríguez Muñoz; el primero junto a Joaquim Garriga Riera en la Universitat de Girona (2010) y Luis Villanueva Bartrina en la Politécnica de Catalunya (2011), el segundo junto a Francisco Javier de la Plaza Santiago (2002) y Juan Miguel Aguado Terrón (2008), ambas leídas en la Universidad de Murcia. El resto de profesores han codirigido una tesis (Tabla V).

Tabla V. Codirección de tesis doctorales

\begin{tabular}{|c|c|c|}
\hline Año & Tesis & Codirectores \\
\hline 2012 & 7 & $\begin{array}{l}\text { Agra Pardiñas, M. J. y Marín Viadel, R. } \\
\text { González Díez, L. y Pérez Cuadrado, P. } \\
\text { Navarro Segura, M. I. y Esteban Maluenda, A. M. } \\
\text { Ramos Espejo, A. y Fernández Barrero, M. A. } \\
\text { Ruiz Collantes, F. J. y Capdevila, J. } \\
\text { Reyes Téllez, F. y Menéndez Robles, M. L. } \\
\text { Santé Riviera, I. y Crecente Maseda, R. }\end{array}$ \\
\hline 2010 & 5 & $\begin{array}{l}\text { Espejo, T.; Osakar, P. } \\
\text { Ferreiro Prieto, J. I., Martí Ciriquián, P. y Oliva Meyer, J. } \\
\text { Iturrate Cárdenes, F. y González Antón, J.; } \\
\text { Lerma García, J. L. y Hernández López, D.Regot Marimon, J. y Garriga Riera, J. }\end{array}$ \\
\hline 2011 & 4 & $\begin{array}{l}\text { Arana Martínez, J. M. y García Meilán, J. J. } \\
\text { Marzal Felici, J. J. y Domenech Fabregat, H. } \\
\text { Prat Caros, J. y Grau Rebollo, J. } \\
\text { Regot Marimón, J. y Villanueva Bartrina, L. }\end{array}$ \\
\hline 2007 & 4 & $\begin{array}{l}\text { Méndez Baiges, M. T. y Garmelo Díez, M. I. } \\
\text { Montesinos Blanco, A. y Navarrete Tudela, A. } \\
\text { Sánchez Méndes, M. y Van den Eynde Collado, C. } \\
\text { Vilar Roca, G. y Ramos Irizar, A. }\end{array}$ \\
\hline 2008 & 4 & $\begin{array}{l}\text { Montero Díaz, J. y Calvo Serraller, F. } \\
\text { Moreiro González, J. A. y Robledano Arillo, J. } \\
\text { Rodríguez Muñoz, J. V. y Aguado Terrón, J. M. } \\
\text { Saavedra Tortosa, G. y Martínez Corral, M. }\end{array}$ \\
\hline 2002 & 3 & $\begin{array}{l}\text { Casado de Amezua Vázquez, J. y Calatrava Escobar, J. A } \\
\text { Gastaminza, F. del y Reig García, R. } \\
\text { Rodríguez Muñoz, J. V. y Plaza Santiago, F. J. de la Valle }\end{array}$ \\
\hline 2000 & 2 & $\begin{array}{l}\text { Bernal Rodríguez, M. y Vázquez Medel, M. A. } \\
\text { Romero Santamaría, A. y García Guata, M. }\end{array}$ \\
\hline 2001 & 2 & $\begin{array}{l}\text { Molina Alarcón, M. y Peiró López, J. B. } \\
\text { Pujol Ramo, J. y Capilla Perea, P. }\end{array}$ \\
\hline 2003 & 2 & $\begin{array}{l}\text { Calle, R. de la y Esteban Fernández, P. } \\
\text { Tomás Sanmartín, A.; Lita Sáez, F. }\end{array}$ \\
\hline 2004 & 2 & $\begin{array}{l}\text { Calle R. de la y Aliaga Morell, J. } \\
\text { Fernández Polanco, A. y Perea González, J. }\end{array}$ \\
\hline 2005 & 2 & $\begin{array}{l}\text { Blázquez Pérez, J. y Olmos Romera, R. } \\
\text { Calle de la Calle, R. de la y Pedraza Martínez, P. }\end{array}$ \\
\hline 1999 & 1 & Herranz Pazcual, Y. y Calle, R. de la \\
\hline 2006 & 1 & García Fraile, D. y Rodríguez de la Flor, F. \\
\hline
\end{tabular}




\section{CONCLUSIONES}

La fotografía como elemento de comunicación, artístico, o científico está resultando un campo de investigación cada vez más abierto, como lo demuestran los resultados del estudio. Utilizada en diversos campos, facultades e instituciones científicas, se trata de un documento social, informativo y científico de primer orden. Además, como soporte documental viene a dar testimonio de una época, de ahí la importancia que tiene su seguimiento, estudio e investigación en la Universidad.

El estudio y análisis de las tesis doctorales permite a la comunidad científica situarse frente a una información que aún sabiendo que existe no se valora en su conjunto, de ahí la importancia de este tipo de trabajos. Reunir, ordenar y clasificar las tesis doctorales de fotografía aporta también un valor a las instituciones donde se defendieron, también a sus directores y codirectores, así como a los departamentos. Además, marca las líneas de trabajo e investigación de algunas facultades, que sin ser mayoritarias, al menos, consideran el tema de la fotografía entre sus campos de estudio.

Una vez analizados los datos podemos concluir que la producción de tesis en el periodo estudiado (1976-2012) es de 275 trabajos, distribuidos en cuatro grandes áreas: Bellas Artes, Ciencias, Comunicación y Humanidades. La valoración global es que los trabajos de investigación sobre fotografía van en aumento, y que se encuentran muy repartidos en las universidades, facultades y departamentos. Así mismo son muchos los directores y se constata también el aumento progresivo de las codirecciones.

La universidad española con mayor número de tesis sobre fotografía es la Complutense (49), seguida de la Universitat Barcelona (26), la Politècnica de Valencia (22) y la de País Vasco (21). Las facultades con mayor producción son las de Bellas Artes de la Complutense (25), de la Politècnica de Valencia (20), Universidad de Barcelona (20), y Ciencias de la Información de la Complutense (20). En cuanto a los departamentos, aunque en conjunto son los de Historia del Arte los que han leído más tesis (44), figura en primer lugar el de Comunicación Audiovisual y Publicidad I de la Complutense (9), después el de Historia del Arte y Bellas Artes de la Universidad de Salamanca (8) y en tercer puesto el de Bellas Artes de Castilla-La Mancha (7).

Por lo que respeta a los contenidos, las tesis más numerosas son las de Arte (76) junto a las de Comunicación y Documentación (80), seguidas de las de Humanidades (45). Es significativo el número de tesis sobre autores (38), en porcentaje similar a las científicas, técnicas o tecnológicas (36).

Los profesores encargados de las asignaturas relacionadas con la materia tienen diversa formación académica, generalmente relacionada con los centros donde se imparten estudios de Bellas Artes e
Historia del Arte, Comunicación, Documentación y otros de carácter técnico. Salvo excepciones, son pocos los que han dirigido muchas tesis, destacando Joaquín Perea González con 7, seguido con Ramón de la Calle con 6. De los 251 directores, 213 han dirigido o codirigido un trabajo.

\section{NOTAS}

[1] Asignatura: Documentación Fotográfica e Iconográfica.

[2] Asignaturas: 1. Problemas de recuperación y gestión del patrimonio fotográfico. Casos prácticos, 2. Problemas técnicos en la recuperación, conservación y reproducción en el ámbito del patrimonio fotográfico, 3. Problemas metodológicos. Historia de la fotografía, 4. Fotografía. Problemas históricos.

\section{BIBLIOGRAFÍA}

Barthes, R. (1994). La cámara lúcida. Notas sobre la fotografía. Barcelona: Paidós Comunicación, p. 31.

Castillo, A.; Xifra, J. (2006). Investigación bibliométrica en las tesis doctorales españolas sobre relaciones públicas (1965-2005). Análisis, vol. 34, 141-161.

Delgado, E.; Torres, D.; Jiménez, E.; Ruiz-Pérez, R. (2006). Análisis bibliométrico y de redes sociales aplicado a las tesis bibliométricas defendidas en España (1976-2002): temas, escuelas científicas y redes académicas. Revista Española de Documentación Científica, 29 (4), 493-524.

Embid Irujo, A. (ed.) (2002). Legislación universitaria. Normativa general y autonómica. Madrid: Tecnos, p. 1056.

Fontcuberta, J. (1983). Apéndice: notas sobre la fotografía española. En: Newhall, B. Historia de la fotografía. Desde sus orígenes hasta nuestros días. Barcelona: Gustavo Gili.

Freund, G. (1999). La fotografía como documento social. Barcelona: Gustavo Gili, p. 208.

Fuentes i Pujol, M. E.; González Quesada, A. (2002). Tesis doctorales en España en información y documentación, 1976-2001. En Morán Suárez, Ma A.; Rodríguez López, M. C. (Coord.) La documentación para la investigación. Homenaje a José Antonio Martín Fuertes. León: Universidad de León.

Jones, D. E.; Barí I Queralt, B. (2000). Investigación sobre Comunicación en España. Aproximación bibliométrica a las tesis doctorales (1926-1998). Barcelona: ComCat, p. 152.

López Yepes, J.; Fernández Bajón, M. T.; Orera Orea, L., Sánchez Vigil, J. M. (2008). Criterios para la evaluación de tesis doctorales. Revista General de Información y Documentación, 18, 1, pp. 293-322.

Marcos Recio, J. C.; Martínez Pestaña, M. J.; Blasco López, M. F. (2012). Producción y dirección de tesis doctorales sobre publicidad en la universidad española (1971-2010). Revista Española de Documentación Científica, 35 (3), 433-452. http:// dx.doi.org/10.3989/redc.2012.3.890

Ministerio de Ecuación y Ciencia (2007). Real Decreto del Ministerio de Educación y Ciencia 1393/2007, de 
29 de octubre, por el que se establece la ordenación de las enseñanzas universitarias oficiales. $B O E, n^{\circ}$ 260 (30/10/2007), 44037-44048.

Moralejo Álvarez, M. R. (2000). Las tesis doctorales en las universidades españolas, Control bibliográfico y acceso. Revista General de Información y Documentación, vol. 10 (1), 235-243.

Moreiro González, C. J. (2010). La cooperación internacional y europea para rescatar la memoria audiovisual. Revista General de Derecho Europea, vol. 22, 1-33.

Repiso, R.; Delgado, E.; Torres, D. (2007). Análisis bibliométrico de la producción española de tesis doctorales sobre cine. En: Bort Gual, I.; García Catalán, S.; Martín Núñez, M. (editores). Actas del IV Congreso Internacional sobre análisis fílmico. Nuevas tendencias e hibridaciones de los discursos audiovisuales en la cultura digital contemporánea. Castellón: Universitat Jaume I.

Repiso, R.; Torres, D.; Delgado, E. (2011a): Análisis bibliométrico y de redes sociales en tesis doctorales españolas sobre televisión (1976-2007). Comunicar, vol. 37 (XIX), 151-159. http://dx.doi. org/10.3916/C37-2011-03-07

Repiso, R.; Torres, D.; Delgado, E. (2011b). Análisis de la investigación sobre Radio en España: una aproximación a través del análisis bibliométrico y de Redes Sociales de las tesis doctorales defendidas en España entre 1976-2008. Estudios sobre el mensaje periodístico, vol. 17 (2), 417-429.

Riego, B. (1993). La fotografía en la enseñanza universitaria: hacia un futuro sin apenas pasado. Jornadas de estudio Nueva Lente. Inicio y desarrollo de la fotografía de la creación en España. pp. 203214. Madrid: Nueva Lente.

Sánchez Vigil, J. M. (2006). El documento fotográfico. Historia, usos, aplicaciones. Gijón: Trea, p. 404.

Sanz Casado, E.; Filippo, D.; García Zorita, J. C.; García, Preiddy E. (2011). Observatorio IUNE: una nueva herramienta para el seguimiento de la actividad investigadora del sistema universitario español. Bordon. Revista de pedagogía, vol. 63, (2), 101-116.

Vázquez Casillas, F. (2008): La historia y la fotografía en España (1975-1999). Estado de la cuestión. Actas del Tercer Congreso de Historia de la Fotografía, pp. 215-227. Zarautz: Photomuseum.

Vega, C. (2007). La fotografía en la Universidad: Una historia con futuro. Actas del II Congreso de Historia de la Fotografía. pp. 66-71, Zarautz: Photomuseum. 


\section{Anexo. Tesis doctorales por año, director, universidad y autor}

Fuentes: Base de datos Teseo (Ministerio de Educación, Cultura y Deporte); Portal Dialnet (Universidad de la Rioja); Catálogo Cisne (Universidad Complutense); Rebiun (Red de bibliotecas Universitarias); Centro Virtual Cervantes; Jones, D. E. y Barí i Queralt, B. (2000).

\section{año*director*universidad*autor}

ac (A Coruña), ab (Autónoma de Barcelona), al (Alicante), am (Autónoma de Madrid), ub (Barcelona), cd (Cádiz), ca (Cantabria), c3 (Carlos III de Madrid), cm (Castilla La-Mancha), cs (Católica de San Antonio), ucm (Complutense), ex (Extremadura), gi (Girona), gr (Granada), ib (Illes Balears), ja (Jaén), jI (Jaume I), la (La Laguna), le (León), II (Lleida), ma (Málaga), mh (Miguel Hernández), mu (Murcia), uned (Nacional de Educación a Distancia), na (Navarra), oc (Oberta de Catalunya), ov (Oviedo), pg (Palmas de Gran Canaria), pv (País Vasco), pc (Politècnica Catalunya), pm (Politécnica de Madrid), pva (Politècnica de Valencia), pf (Pompeu Fraba), pn (Pública de Navarra), rl (Ramón Llull), rj (Rey Juan Carlos), rv (Rovira i Virgili), sa (Salamanca), ceu (San Pablo Ceu), sc (Santiago de Compostela), se (Sevilla), va (Valencia), vll (Valladolid), vi (Vigo), uz (Zaragoza).

1976*torroja menéndez, j.m.*ucm*garcía pelayo,j.m. 1977*floristán imízcoz,a.p.*na*lizarraga lezaun, m.a. 1977*oliva molina, j.*va*pardo casado, $\mathrm{m}$. 1980*lara garcia, a.*ucm*villafañe gallego, j. 1981*jiménez sandín, f.j.*pm*hernán gómez, m.á. 1981*ras oliva, g.*pc*roure fernández, f. 1983*sánchez-mesa martín, d.*ma*coloma martín, i. 1984*pardo casado, m*al*bonmati madro, a.l. $1986 *$ cebrián herreros, m.*ucm*alonso erausquin, m. 1986*lara garcía, a.*ucm*perea gonzález, j. 1986*predo pico, e.*ab*ledo andion, $\mathrm{m}$. 1987*gonzález de durana, j.*pv*susperregui, j.m. 1987*legorburu escudero,p.*pv*barandiaran, $\mathrm{m}$. 1987*prado pico, e.*pv*bilbao fullaondo, j.m. 1988*cabañas bravo, j. m.*uz*pérez lizano forns, m. $1988 *$ calle, r. de la*va*mira pastor, e. 1988*gómez molina, j.j.*sa*menor salas, m. 1989*aguilera pedrosa, a.*ub*caja lópez, f. 1989*blasco carrascosa, j.a.*pva*alcalá mellado, j.r. 1989*garcerán piqueras, r.*ucm*bueno thomas, a. 1989*mayné torras, j.*ub*carbó perseguer, e. 1989*sánchez carralero, r.*sa*sierra puparelli,v. 1990*garcía-nobleja, j.j.*na*peltzer alaman, g.m. 1990*noblejas linier, j.j.*pv*esparza estaun, j.r. 1990*zubiaur carreño, f.j.*na*rodríguez meza, v.m. $1991 *$ becker, w.*ucm*río santos, g. $1991 *$ caja lópez, f.*ub*tapias gil, m.d. $1991 *$ carbó perseguer, e.l.*ub*de castro fondevila, a. $1991 *$ martín rodríguez, f. g.*la*vega de la rosa, c. $1991 *$ silvestre visa, m.*pva*ibáñez magraner, e. $1992 *$ cebrián, m.*ucm*rodríguez merchán, e. $1992 *$ garcía melero, j.e.*uned*bastida calle, m.d. 1992*pérez mulet, f.*cd*garofano sánchez, r. 1992*redondo gálvez, j.a.*na*vidal-quadras, j.a. 1993*aciron royo, r.*la*gutiérrez rodríguez, o. 1993*lara garcía, a.*ucm*casajus quiros, m. c. 1994*garcía melero, j.e.*uned*pando despierto, j. 1994*gonzález garcía, a.*sa*jiménez brobeil, j.l. 1994*iglesias rodríguez, h.*pm*fernández barredo, y. 1994*lópez de sagredo, f.*pm*gómez-elvira, m.a. 1994*martín gonzález, f.*ucm*caballo ardila, d. 1994*perea gonzález, j.*ucm*gómez redondo, m.j. 1994*vázquez medel, m.a.*sa*domínguez m.l. 1994*yturralde lópez, j.m.*pva*armand buendía, I. 1995*bados iparraguirre, a.*pv*garcía gonzález, m.á. $1995 *$ calle r. de la*pva*monleón pradas, e. e. 1995*garcía garcía, f.*ucm*bañuelos capistran, j.i. 1995*jiménez huertas, i.*pv*elorza ibáñez gauna, c. 1995*ledo andión, m.*ab*furió sancho, j. m. 1995*leyra soriano, a.m.*ucm*vargas martinto, r. 1995*lópez yepes, j.*ucm*sánchez vigil, j. m. 1995*perea gonzález, j.*ucm*castelo sardina, l. 1995*rekalde izaguirre, j.*pv*calderón blanco, r. 1996*caja lópez, f.*ub*inserser brufau, e. 1996*gómez molina, j.j.*le*tranche fernández, e. 1996*gonzález garcía, á.*cml*fernández martínez, h. 1996*gonzález requena, j.*am*baena fíaz, f. 1996*lópez garcía, a.*va*ortiz gil, a. 1996*pérez cortés, r.*sa*gonzález romero, d. 1996*romero rubio, a.*ucm*san martín pascal, m.á. 1996*sánchez gzález,s.*ucm*marcos molano, m.m. $1997 *$ castro fernández, x.a.*uz*sendón trillo, m. 1997*garcía melero, j.e.*uned*alonso martínez, f. 1997*ledo andión, m.*sc*sousa, j. p. 1997*pascual pérez, c.*sa*gonzález fernández, r.a. $1997 *$ valle garagorri, a.*ucm*gómez isla, j. 1998*aznar vallejo, j.f.*pva*acosta torres, j.f. 1998*barrio alonso, a.*ca*riego amezaga, b. 1998*caja López, f.*ub*alberich pascual, j. $1998 *$ caja lópez, f.*ub*terré alonso, I. 1998*caja lópez, f.*ub*torres martínez, j. m. 1998*esparza estaún, j.r.*pv*fernández bañuelos, j.i. 1998*garcía gainza, c.*na*domeño martínez, m.a. 1998*garcía gainza, c.*na*latorre izquierdo, $j$. 1998*herrero gómez, j.m.*la*ruiz pacheco, m.m. 1998*jiménez martín, a.*gr*jiménez martín, c. $1998 *$ moya valgañón, a.*pv*sixto cesteros, r. 1998*sánchez carralero lópez, r.*sa*carrera, v. $1998 *$ toran pelaez, e.*ucm*moreno de carlos, d. 1999*aznar vallejo, j.f.*la*swinnen johan, m. 1999*cantarellas camps, c.*ib*mulet gutiérrez, m.j. 1999*galante gómez, f. j. *la*garcía garcía, j. 
1999*hernáiz blázquez, j.i.*ucm*martín lópez, a.m. $1999 *$ herranz, y.; calle, r. de la*vi*noguerira, c. 1999*ledo Andión, m.*ab*baeza gallur, j. 1999*prado pico, e.*ab*hernández gómez, j.b. 1999*ruíz rico morón, j.*gr*valero martín, e. 1999*salinas gonzález, f.j.*pm*velilla lucini, c. 1999*urabayen cascante, m.*na*sanz garrido, b. 1999*zubiaur carreño, f.j.*na*martín larumbe, c. 2000*barañano, k.*pv*garcía landeras, p.j. 2000*bernal, m.; vázquez, m.a.*sa*belenguer, m. 2000*caminos marcet, j.m.*pv*gutiérrez coba, I. 2000*galera andreu, p.a.*gr*pérez miñano, c. 2000*giménez morell, r.*pva*gómez frías, r. 2000*gonzález antón, f.j*la*teixeira ribeiro, I. a. 2000*hernández pérez, a.*c3*robledano arillo, j. 2000*lópez cuervo, s.*pm*fernández molowny, a. 2000*perea gonzález, j. *ucm*munarriz ortiz, j, 2000*rodríguez dieguez, j.I.*sa*linares linares, p. 2000*romero, a.; garcía guata, m.*uz*espa lasaosa, v. $2001 *$ bilbao fullaondo, i.*pv*ruíz san miguel, f.j. $2001 *$ galindo gálvez, j.*pva*cueto lominchar, j.l. $2001 *$ garcía santos, r.*pn*santamaría peña, j. 2001*gonzález alonso, s. *pm*gonzález echeverría, r. 2001*latorre izquierdo, j.*na*garay albujar, a. 2001*molina, m.; peiro, j.b.*pva*martínez , c. 2001*parralo dorado, m.*ucm*lópez raso, p. 2001*penousal, f. j.; romero, j.j.*Il*carballal, a. 2001*perea gonzález, j.*ucm*legido garcía, m.v. 2001*pujol, j.; capilla, p.*pc*martínez verdú, f.m. $2001 *$ vélez cea, m.*gr*morillas puente, i. 2002*casado, j.; calatrava, j.a.*gr*gómez-blanco, a.j. $2002 *$ chavete rodríguez, j.*vi*arrillaga chopieta, j. $2002 *$ garriga escribano, c.*urv*gallego paz, r. $2002 *$ martínez salmean, m.l.*ucm*benito oterino, a. 2002*pena rodríguez, a.*vi*lens leiva, $j$. 2002*rodríguez, j.v.; plaza, f. j.*um*manzanera, m. $2002 *$ valle, f.; reig garcía, r.*sa*bobo márquez, m.l. 2003*anta félez, j.l.*cm*lara lópez, e.I. $2003 *$ calle, r. de la; esteban, p.*pva*prado, m.e. 2003*carrera suárez, m.i.*ov*torres forciniti m. a. 2003*Fernández díez, f.*ab*correa larrain, m.m. 2003*fimia fil, a.*mh*ulibarrena estévez, m. 2003*hernaiz blázquez, j.i.*ucm*sánchez garre, m.n. 2003*humanes humanes, m. I.*sa*gomes golzio, d. 2003*laguillo, m.*ub*borges vaz dos reis, e. t. 2003*león gross, t.*ma*gonzález aranda, f. 2003*martín francés, a.*ucm*labastie, f. 2003*pablos coello, j.m.*la*magaña toledano, j. c. 2003*ramírez domínguez, j.a.*am*bravo López, I. 2003*ranch sales, e.*al*mora Santacruz, I. 2003*ruiz rodríguez, a.á.*gr*salvador benítez, a. 2003*tomás, a.; lita sáez, f.*pva*miralles, a.m. 2003*vilches, I.*ab*alba Gutiérrez, g. 2004*aliaga morell, j.*pva*benlloch serrano, j. 2004*aliaga morell, j.*pva*ortega aznar, a. t. 2004*arocena badillos, c.*pv*villota toyos, g. 2004*azpeitia burgoz, a.*uz*irala hortal, m. p. 2004*calle, r. de la; aliaga, j.*pva*vera casas, f. 2004*carbó perseguer, e.I.*ub*portolés brasó, f. 2004*casajús quirós, m.c.*ceu*santiago mateos, m.a. 2004*caselles costa, v.*pf*verdera ribas, $j$. 2004*fernández, a.; perea gzlez, j.*ucm*vélez,g.m. 2004*huertas torrejón, m.*ucm*meseguer mayoral, r. 2004*lara garcía, a.*ucm*armengol antonio, g. 2004*manzanera niñirola, m.*um*vázquez, j.f. 2004*molina alarcón, m.*pva*cremades juan, m. a. 2004*montesinos blanco, a.* ${ }^{*} \mathrm{ml} *$ lin kan, s. 2004*perea gonzález, j.*ucm*ruiz mollá, i. 2004*reyero hermosilla, c.*am*Alonso laza, m. 2004*saura ramos, p. a.*ucm*morgado aguirre, b. 2004*silvestre visa, m.*pva*march ten. a. 2004*soler sanz, f.*pva*maestre lópez-salazar, r. 2004*vilá matínez, a.*ub*pastó aguilá, c. 2005*blázquez, j.;olmos roquera, r.*am*gonzález, s. 2005*brisset martín, d.*ma*garnelo díez, m. i. 2005*buzo rey, m. j.*ub*renobell santarén, v. $2005^{*}$ de la calle, r.; pedraza, p.*va*cancer, j.r. 2005*esteve ramírez, f.*ucm*caballo méndez, d. 2005*fernández martínez, h.*cm*martínez, c. 2005*garrido sánchez, c.*ucm*martínez, j.m. 2005*lópez lita, R. *jI*soler campillo, m. 2005*machado baldasano, f.*mh*shulte, s. 2005*marzal felici, j.*jI*aguilar garcía, j. a. $2005 *$ marzal felici, $j . * j I *$ domenech fabregat, $h$. 2005*navarrete tudela, a.*cml*yuste pescador, j. r. 2005*ochotorena, j.m.*na*alcolea rodríguez, r.a. 2005*perea gonzález, j.*ucm*trobat bernier, r. 2005*pérez jofre, i.*vi*fernández prada, e. 2006*cazalla pinero, j. m.*pva*tejo veloso, c. 2006*esparza estaún,j.r.*pv*miguel sáez de, a. 2006*fernández martínez, h.*cml*oleza simo, j. 2006*garcía fraile,d.; rodríguez ,f.*sa*maillo, f. 2006*morey farré, m.*ub*bofill garcia amorena, c. 2006*ros garcía, j.*um*garcía belchí, m. 2006*terrasa, j.*pv*romo mellid, m. $2006 *$ velilla lon, c.*ub*cantalozella planas, j. 2006*villegas garcía, m.*cml*frías salazar, v. m. 2007*barroso, j, *ucm*cuevas martín, j. $2007 *$ cirlot valenzuela, l.*ub*rueda fajardo, s. $2007 *$ espina barrio, a.b.*sa*de andrade quintas, g. 2007*huertas torrejón, m.*ucm*gonzaléz jiménez, n. 2007*idoate iribarren, j.*pv*cabral machado, g. 2007*ledo andión, m.*sc*ribao fernández, r. 2007*marcos molano, m.*ucm*sáez pedrero, a. 2007*mendez, m.t.; garnelo, m.i.*ma*garcía, n. 2007*montesinos, a.; navarrete t, a.*cm*paz, t.de j. 2007*sánchez, m.; van den eynde,c.*ucm*moreno, m. 2007*tapias gil, m.d.*ub*moura possa, c. m. 2007*vilar roca, g.; ramos irizar, a*ab*ugarte, s.e. 2008*aliaga espert, j.v.*pva*sentamans gómez, t. 2008*álvarez gila, o.*pv*manfredi, $\mathrm{m}$. 2008*arnaiz gómez, a.*pv*claver sanz, v. m. 2008*casajús quirós, m.c.*ceu*guerrero gonzález, b. 2008*esparza estaún, j.r.*pv*parejo jiménez, n. 2008*graña romay, m.*pv*maldonado bautista, j.o. 2008*laguillo menéndez, m*ub*zuzunaga, m. 
2008*monleón pradas, e.e.*pva*zarraga llorens, j.m. 2008*montero, j.; calvo, f.*ucm*ortiz-echague, j. 2008*moreiro, j.a.; robledano, j.*c3*torres, n. 2008*palomer mateos, p.*ub*negre buso, $\mathrm{m}$. 2008*pinto molina, m.*gr*doucet, a.v. 2008*reder gadow, m.*ma*ramírez gonzález, j. 2008*rodríguez, j.v.; aguado, j.m.*um*castillo, j.m. 2008*saavedra, g.; martínez, m.*va*martínez, r. 2008*solana suárez, e.*pg*van geeteruyen ibáñez, l. $2008 *$ vega de la rosa, c.*la*tudela caño, p. 2008*zunzunegui díez, s. ${ }^{*}$ pv*elorza insausti, m. 2009*alemán bastarrica, a.*la*arozena bonnet, m.t. 2009*amador carretero, p.*c3*hottinger graig, s. 2009*bueno tomás, a.*ucm*vega borrego, d. 2009*castelo sardina, l.*ucm*santos alguacil, m. 2009*marcos molano, m. del.*ucm* lópez álvarez, a. 2009*monegal brancos, a.*pf*prieto aguaza, a. 2009*pérez mulet, f.*cd*portillo guzmán, f. 2009*perñan rafart, m.*ub*paris romia, g 2009*sendón trillo, m.*vi*garcía garcía, i. 2009*tapias gil, m.d.*ub*guixá frutos, r. 2010*delgado garcía, j.*ja*lara cabeza, j. m. 2010*diego, estrella de*ucm*cruz lichet, v. de la 2010*espejo, t. ; osakar, p.*gr*campillo garcía, d. 2010*ferreiro, j.i.;martí, p.;oliva, j.*al*pérez, r. 2010*garcía garcía, f.*ucm*gómez lozano, d. 2010*guillén ramón, j.m.*pva*hernández gil, I. 2010*iturrate, f.; gonzález, j.*la*abreu sojo, c.a. 2010*lerma, j.I.; hernández, d.*pva*felipe garcía, b. 2010*martín martínez, j.v.*mh*albalate gauchía, i. 2010*marzal felici, j.*jI*peset ferrer, j. p. 2010*pfeiffer, m.*pf*sánchez cardona, a. p. 2010*regot, j.; garriga riera, j.*gi*moliner milhau, $x$ 2010*renard álvarez, s.*va*santamarina campos, p. 2010*sánchez dura, n.*va*lópez sanz, h. g. 2010*sánchez vigil, j.m.*ucm*olivera zaldua, m. 2010*tapias gil, m.d.*ub*redondo arolas, m. 2010*tapias gil, m.d.*ub*freixa font, p. 2011*almarcha núñez, m.e.* ${ }^{*}{ }^{*}$ espinosa, n. 2011*arana, j.m.; garcía meilán, j.j.*sa*gorsillo, f. 2011*ardevol piera, e.*ob*gómez cruz, e. $2011 *$ esquerro noblejas-garcía, m.*ac*pomin, f. 2011*garcía pañella, o.*el*fonseca escuderi, d. $2011 *$ guasch ferrer, a. m.*ub*grigoriadu, e. 2011 *huerta ramón, r.*va*huerta ramón, r. $2011 *$ marti aris, c. $*$ pc*ariza castri, f. 2011*martín francés, a.*ucm*casula oliver, c. 2011*martínez rodríguez, a.*sa*torralva forero, a.m. 2011*marzal, j.j.; domenech, h.*jI*arroyo, l.b. $2011 *$ orella martínez, j.l.*ceu*rigaud lojon, g.a. 2011*panera cuevas, f.j.*sa*albarrán diego, j. 2011*pérez rodrigo, d.*pva*pérez fernández, j.r. 2011*plaza santiago, f.*vll*moreno cantero, r. 2011 *prat caros, j.; grau rebollo, j.*rv*gonzález, p. 2011*regot, j.; villanueva, l.*pc*bover tanya, a. $2011 *$ rodríguez de las heras, a.*c3*heras, b. de las $2011 *$ sala garcía, t.*ub*fernández rius, n. 2012*abad tejerina, m.j.*ucm*torrado zamora, I. 2012*agra pardiñas, m.j.; marín, r.*gr*mesías, j.m. 2012*barragán roríguez, j.m.*ub*viñuales lera, d. $2012 *$ calzado almodóvar, z.*ex*vilches cerrato, m. 2012*carbo perseguer, e.l.*ub*duarte de sousa, a. 2012*castelo sardina, I.*ucm*corrales crespo, e. 2012*garci santos, a. *pm*santonja jiménez, r. 2012*gonzález. I.; pérez, p.*ceu*heredia, a. 2012*ibañez fanés, f.*ab*gonzález garcía, a. 2012*mancera martínez, m.f.*sa*falcón neyra, a. 2012*marchán fiz, s.*uned*días neves, e. 2012*navarro, m.i.; esteban, a.m.*la*medina, g. 2012*panera cuevas, f.j.*sa*corkovic, I. m. 2012*pastor cubillo, m.b.r.*pva*heras evangelio, d. 2012*ramos, a.; fernández,m.a.*sa*rguez, m.del m. 2012*reyes, f.; menéndez robles, m.I.*rj*díaz pena, r. 2012*ruiz collantes, f.j.; capdevila, j.*Il*visa, m $2012 *$ santé, i. ; crecente, r.*sc*corbelle rico, e. 2012*tapias gil, m. d.*ub*pardo saínz, r. 2012*vázquez medel, m.a.*sa*luzón toro, c. 